\title{
actions de l'environnement polaire sur la digue en enrochements, support de la piste d'aviation en Terre Adélie (Antarctique)
}

\author{
polar environment actions on the rubble mound breakwater, \\ support of the Terre Adelie airfield (Antarctica)
}

\author{
J.-F. REGRETTIER \\ Ingénieur \\ Centre de Recherche et Développement du Bureau Veritas" \\ Laboratoire de Tectonophysique *
}

Rev. Franç. Géotech. n 53 , pp. 55-65 (octobre 1990)

\section{Résumé}

Une digue en enrochements de $1100 \mathrm{~m}$ de long sur laquelle doit reposer une chaussée permettant l'atterrissage et le décollage d'avions de type Transall ou Hercules LC 130 est en cours de construction dans l'archipel de Pointe Géologie en bordure du continent antarctique.

Le projet et sa réalisation sont décrits. On étudie l'action de la houle et de l'environnement spécifique aux régions polaires (basses températures, glace) sur l'ouvrage, en particulier le dimensionnement de la carapace, la congélation de l'eau de mer entre les blocs rocheux et la gélifraction des enrochements.

\section{Abstract}

Construction of a rubble mound breakwater, $1100 \mathrm{~m}$ large, which will support the runway for Hercules LC 130 or Transall aircrafts is in progress in Terre Adelie.

The project is described. Action of waves, swell and specific aspects of polar engineering (low temperatures, ice) are studied, particularly the armour design, freezing of seawater within the structure and frost shattering of rock blocks. 


\section{PRÉAMBULE}

Le développement de la recherche scientifique en Antarctique nécessite une logistique de plus en plus puissante. Depuis le débarquement de Dumont d'Urville sur la côte de Terre Adélie en janvier 1840 (fig. 1), à proximité de la base française qui porte son nom, le transport de vivres, de matériel et de personnel se fait par voie maritime. En raison des conditions climatiques hivernales extrêmement sévères, les travaux, en particulier la construction de nouveaux bâtiments et laboratoires, ne peuvent se faire qu'en été.

L'extension de la banquise qui ne disparaît qu'en décembre limite laccès par la mer à une courte période de deux mois et demi (mi-décembre, janvier, février). De plus, la France a un projet ambitieux: la réalisation d'une base sur le plateau antarctique englacé à plus de $1000 \mathrm{~km}$ de la station actuelle (Dôme C). Pour ces raisons, une desserte aérienne est devenue indispensable. Le premier avion pourrait atterrir à la fin du mois d'octobre et le dernier repartir au mois de mars. La durée des campagnes d'été serait ainsi doublée et le temps de transport entre l'Australie, terre civilisée la plus proche, (Hobart (Tas- manie), à plus de $2500 \mathrm{~km}$ ), et Dumont d'Urville passerait de 5 jours (par bateau dans le meilleur des cas) à 5 heures! La piste pourra également être utilisée par un avion léger mixte skis-roues de type Twin Otter pour accéder à la future base continentale.

Certains pays bénéficient déjà de ce moyen de transport. Les américains utilisent au printemps des pistes sur la glace de mer pour avions sur roues et en été des pistes sur neige sur l'ice Shelf à Mac Murdo (fig. 1). Sur la côte, laugmentation de température en été perturbe le trafic. Des avions sur ski peuvent également aller à Amundsen-Scott (pôle sud).

Les Soviétiques entretiennent avec de gros moyens une piste de neige compactée à Molodezhnaya. Les argentins et les chiliens possèdent des pistes en terre dans la péninsule antarctique à Arturo Prat Base et Marsh Martin Station.

Pour utiliser l'avion pendant toute la saison estivale, le territoire des Terres Australes et Antarctiques Françaises (TAAF) a choisi de construire une piste en dur qui ne nécessite pas d'avions spéciaux. (Le prix des skis pour un C 130 représente une part importante du coût total de l'appareil). Elle reposera sur une digue maritime en enrochements.

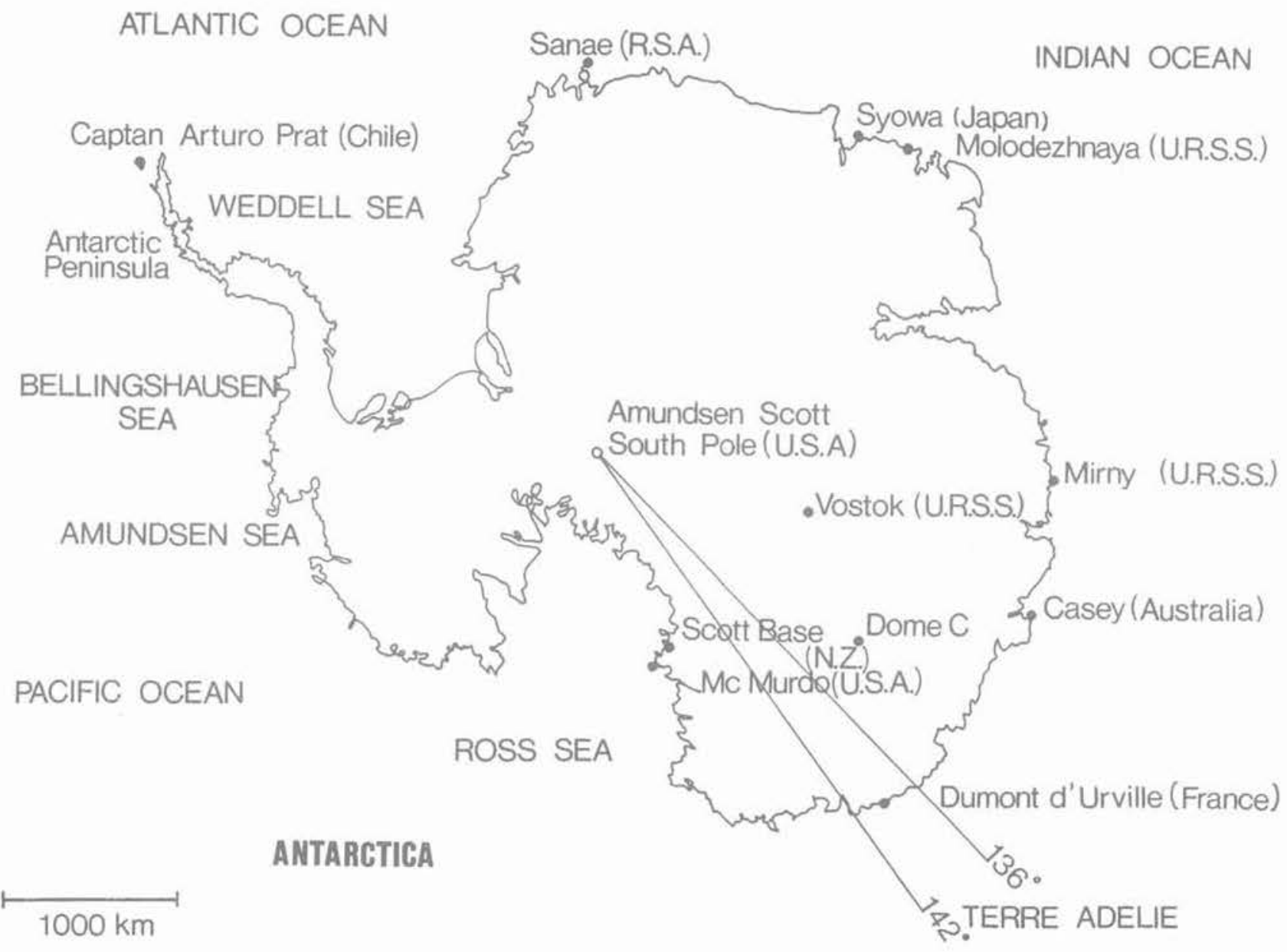

Fig. 1. - Antarctique.

Fig. 1. - Antarctica. 
Parallèlement à la construction de l'ouvrage, un programme d'ingénierie polaire a été lancé. Il doit permettre d'une part, de s'assurer que l'environnement froid n'est pas nuisible à la tenue de l'ouvrage, et d'autre part, de collecter des données qui pourront être utilisées ultérieurement pour des travaux du même type. En effet, à ce jour et à notre connaissance, aucune étude concernant une digue en enrochements en milieu polaire n'a été publiée hormis le rapport de ETTEMA et KENNEDY [5] pour la construction du port de Nome à l'ouest de l'Alaska. $\mathrm{Ce}$ programme est financé en Terre Adélie par la mission de recherche des TAAF et par les Expéditions Polaires Françaises (EPF). Les travaux réalisés en métrople bénéficient du soutien du Ministère de la Recherche et de la Technologie dans le cadre d'un contrat auquel participent le Laboratoire de Géomorphologie du CNRS (Caen), le Laboratoire Central des Ponts et Chaussées, le Laboratoire d'Aérothermique du CNRS (Meudon), les TAAF et le Bureau Veritas.

\section{DESCRIPTION DE L'OUVRAGE PROBLÈMES SPÉCIFIQUES ET MESURES SUR SITE}

Le projet est à l'étude depuis plusieurs années. La solution la mieux adaptée au site est la construction d'une chaussée sur une digue en enrochements reliant entre eux sept îlots de l'archipel de Pointe Géologie (fig. 2) sur lequel est construite la base française. Les sept îlots seront arasés par minage. Les blocs ainsi obtenus permettront de réunir les iles par un remblai et d'obtenir une structure longue de $1100 \mathrm{~m}$, large de $60 \mathrm{~m}$ et dont la plate-forme sera située à $5 \mathrm{~m}$ au dessus du niveau moyen de la mer. L'ouvrage sera constitué approximativement de $50 \%$ de « dur » (iles) et $50 \%$ de remblai. Déblais et remblais nécessitent le transport de $600000 \mathrm{~m}^{3}$ d'enrochements, et l'utilisation de $200 \mathrm{t}$ d'explosif.

Dans ce projet, certains éléments sont favorables :

- louvrage se trouve dans la direction des vents dominants (entre 120 et $140^{\circ} \mathrm{N}$ ) ;

- le fond sous-marin est un substratum rocheux de gneiss. Tassements et poinçonnement des terrains d'assise ne sont donc pas à redouter.

D'autres le sont moins :

- la campagne d'été déjà très courte peut être considérablement réduite par le mauvais temps (possibilité de vents de plus de $200 \mathrm{~km} / \mathrm{h}$ ) ;

- la fin du chantier, prévue pour la fin de l'année 1992, début 1993 peut se voir reportée à l'année suivante.

Dans cette région, la mer est gelée d'auril à décembre. La digue est soumise a la double action de la mer et de la glace. Elle doit résister :

- en été aux vagues, à la houle, à limpact de glaces flottantes ;
- en hiver à la banquise, aux contraintes induites par le changement de phase de l'eau à l'intérieur de l'ouvrage, à la gélifraction des blocs.

Depuis 1987, une digue expérimentale de dimensions $100 \mathrm{~m} \times 60 \mathrm{~m} \times 5 \mathrm{~m}$, construite en 1985, a été instrumentée. De nombreux forages ont été réalisés et équipés de thermistances reliées à des enregistreurs numériques Grant qui stockent les valeurs de la température toutes les heures. Des mesures de houle ont été effectuées à l'aide d'un écho sondeur posé sur le fond. Des mesures des déformations de surface de la digue ont été faites régulièrement ainsi que des carottages pour déterminer l'épaisseur et la salinité de la glace de mer (ENGLER, REGRETTIER [4]).

\section{ACTION DES BASSES TEMPÉRATURES}

Les mesures réalisées dans les forages ont montré que la température de la mer dans la digue était inférieure à - $1,8^{\circ} \mathrm{C}$ dans certaines zones (c'est la température de congélation de P'eau de mer de Terre Adélie dont la salinité est de $35 \mathrm{~g} / \mathrm{l}$ ), mais que dans d'autres zones, elle pouvait osciller suivant les saisons autour de cette température de changement de phase.

Le volume de la glace varie donc dans le temps, le changement de phase se fait avec une variation de $10 \%$ pouvant engendrer de fortes contraintes. Les mesures nous ont permis de suivre l'évolution à court terme (1 année). Afin d'étudier le comportement à plus long terme, nous avons utilisé le programme GELSOL du Laboratoire Central des Ponts et Chaussées qui permet de suivre l'évolution des températures dans un sol connaissant ses caractéristiques et les actions extérieures.

Dans une première phase, nous avons modélisé la digue expérimentale construite dans $5 \mathrm{~m}$ d'eau. Dans une seconde, nous nous sommes intéressés à une partie de l'ouvrage située dans $20 \mathrm{~m}$ d'eau entre les iles du Lion et Pollux (fig. 2)

\section{Digue expérimentale}

Nous avons utilisé un modèle à 3 couches horizontales de $30 \mathrm{~m}$ de large et $12,25 \mathrm{~m}$ de haut. Le niveau de la mer est à la côte 7,25 . Entre la côte 0 et $3,75 \mathrm{~m}$, la couche représente le substratum sous-marin de gneiss. Entre 3,75 et $7,25 \mathrm{~m}$, il s'agit d'enrochements immergés et entre 7,25 et $12,25 \mathrm{~m}$ d'enrochements hors d'eau (fig. 3).

Les conditions initiales (températures aux quatre coins de la structure) ainsi que les conditions aux limites sont données par les mesures et par les conditions climatiques locales : température de l'air, température de l'eau, température de la glace en hiver, température du substratum (la température de l'air sous abri est la moyenne des valeurs de 1986, 1987, 1988). 


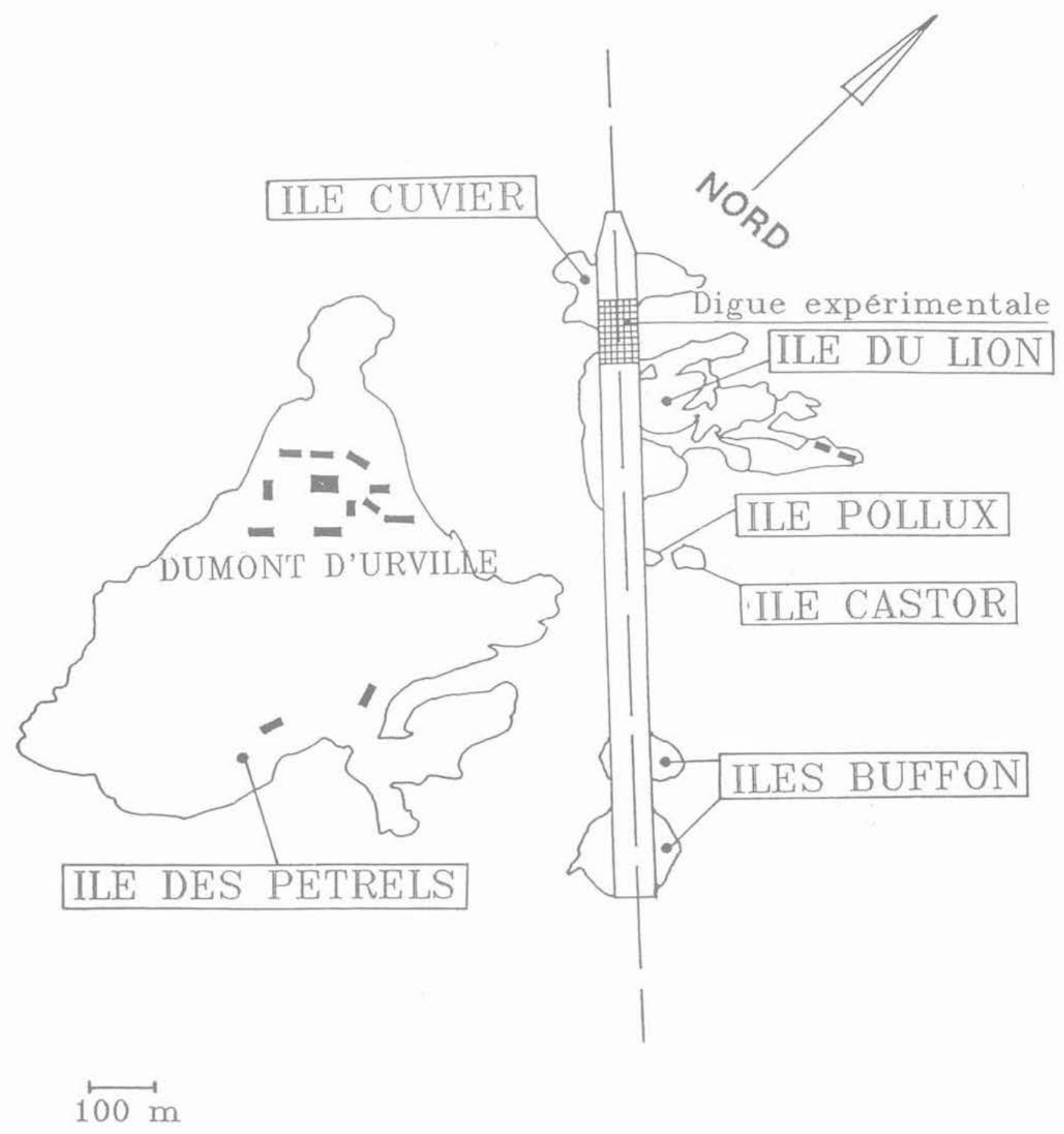

Fig. 2. - Base Dumont d'Urville.

Fig. 2. - Dumont d'Urville Base.

\begin{tabular}{|l|c|c|c|c|c|c|c|c|c|c|c|c|}
\hline Températures & J & F & M & A & M & J & J & A & S & O & N & D \\
\hline Air & $-0,5$ & $-3,1$ & $-8,7$ & $-12,7$ & $-16,2$ & $-15,0$ & $-17,2$ & $-17,2$ & $-14,5$ & $-11,3$ & $-7,5$ & $-2,1$ \\
\hline Eau & $-0,6$ & $-0,6$ & $-1,0$ & $-1,8$ & $-1,8$ & $-1,8$ & $-1,8$ & $-1,8$ & $-1,8$ & $-1,8$ & $-1,8$ & -1 \\
\hline $\begin{array}{l}\text { Substratum } \\
\text { sous talus }\end{array}$ & -2 & -2 & $-2,4$ & $-2,4$ & $-3,6$ & $-3,3$ & $-3,6$ & -5 & -4 & $-3,5$ & $-2,8$ & -2 \\
\hline $\begin{array}{l}\text { Substratum } \\
\text { Centre remblai }\end{array}$ & -4 & -4 & $-3,8$ & -4 & -4 & $-4,5$ & $-5,4$ & -6 & -5 & $-4,4$ & $-4,6$ & -4 \\
\hline
\end{tabular}




\section{MODELE DE REMBLAI EN ENROCHEMENTS}

\begin{tabular}{|c|c|}
\hline \multicolumn{2}{|l|}{ conductivité(gelé): 1,7} \\
\hline densité : 1,73 & $w: 1 \%$ \\
\hline \multicolumn{2}{|c|}{ conductivité(gelé): 3,1} \\
\hline densité : 2,12 & $w: 15 \%$ \\
\hline \multicolumn{2}{|l|}{ conductivité(gelé): 3,5} \\
\hline \multicolumn{2}{|c|}{ conductivité(non gelé): 3,5} \\
\hline densité : 2,6 & $w: 0,1 \%$ \\
\hline
\end{tabular}

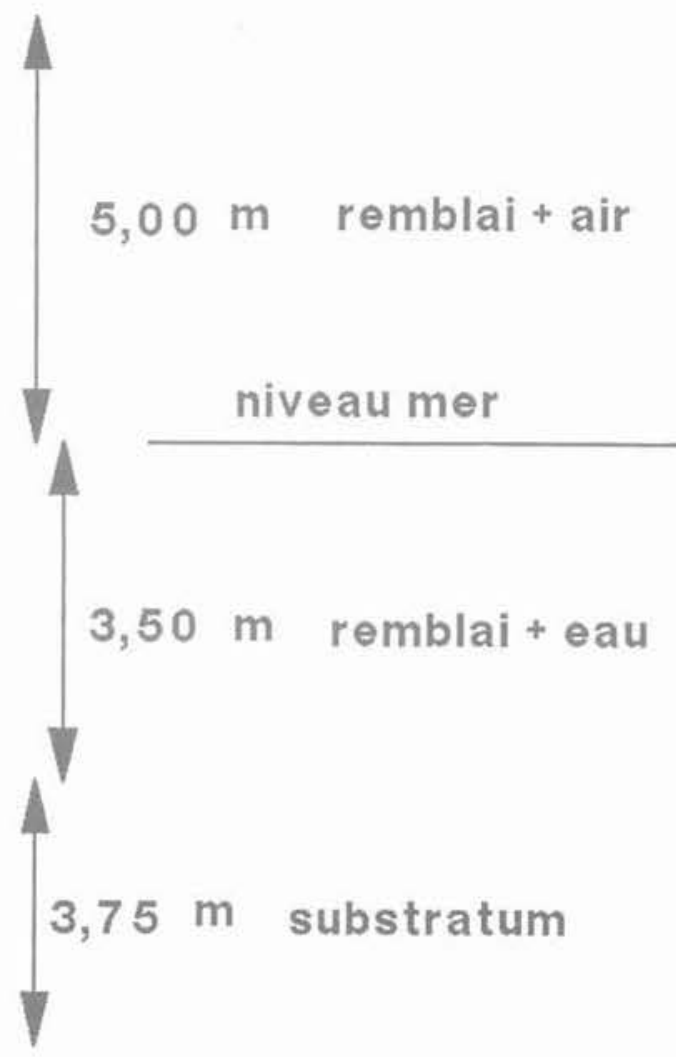

\section{$30,00 \mathrm{~m}$}

\section{coefficient de vide : $30 \%$ conductivités thermiques en $\mathrm{W} / \mathrm{M} . \mathrm{K}$ \\ $w$ : teneur en eau}

Fig. 3. - Modèle de digue pour le programme GELSOL.

Fig; 3. - Breakwater model for GELSOL computer program.

Les caractéristiques du sol sont synthétisées sur la figure 3 . Les conductivités thermiques des enrochements (enrochements + air, enrochements + eau, enrochements + glace) ont été calculées en utilisant le modèle de MICKLEY à partir des conductivités de la roche $(3,5 \mathrm{~W} / \mathrm{mK})$, de l'air $(0,02 \mathrm{~W} / \mathrm{mK})$, de l'eau $(0,5 \mathrm{~W} / \mathrm{mK})$ et de la glace $(2,2 \mathrm{~W} / \mathrm{mK})$ (ANDERSLAND, ANDERSON [1]).

La porosité du gneiss in situ est inférieure à $0,5 \%$. Le coefficient des vides des enrochements est estimé à environ $30 \%$.

Nous n'avons modélisé que la moitié nord-est de louvrage que nous avons considéré comme symétrique. L'abscisse nulle correspond à l'axe de symétrie du remblai. Les résultats sont sous forme de graphiques qui représentent l'évolution des isothermes au cours du temps.

Si on néglige les phénomènes de migration de sels dans la structure (une étude est en cours à partir de données de température, résistivité et capacité enregistrées simultanément dans certains forages), on peut en première approximation considérer que toutes les zones situées sous le niveau de la mer, dont la température est inférieure à $-1,8^{\circ} \mathrm{C}$ sont gelées.

La figure 4 représente les isothermes aux jours 60 , $360,720,1080$ (le jour 0 correspond à la construction du remblai en milieu d'été, l'eau entre les blocs est liquide). On constate qu'après une année, une bonne partie de l'eau s'est transformée en glace et que le volume d'eau restant diminue pour disparaitre complètement après trois années. Lorsque l'on compare ces valeurs calculées aux valeurs mesurées, on constate une bonne concordance excepté à proximité du talus. En effet, par le calcul il reste une frange non gelée dont l'épaisseur est de l'ordre du mètre. Il semble que dans la réalité, cette frange non gelée soit plus importante, en particulier dans la partie nord qui en été est soumise à la houle et donc à une forte con- 


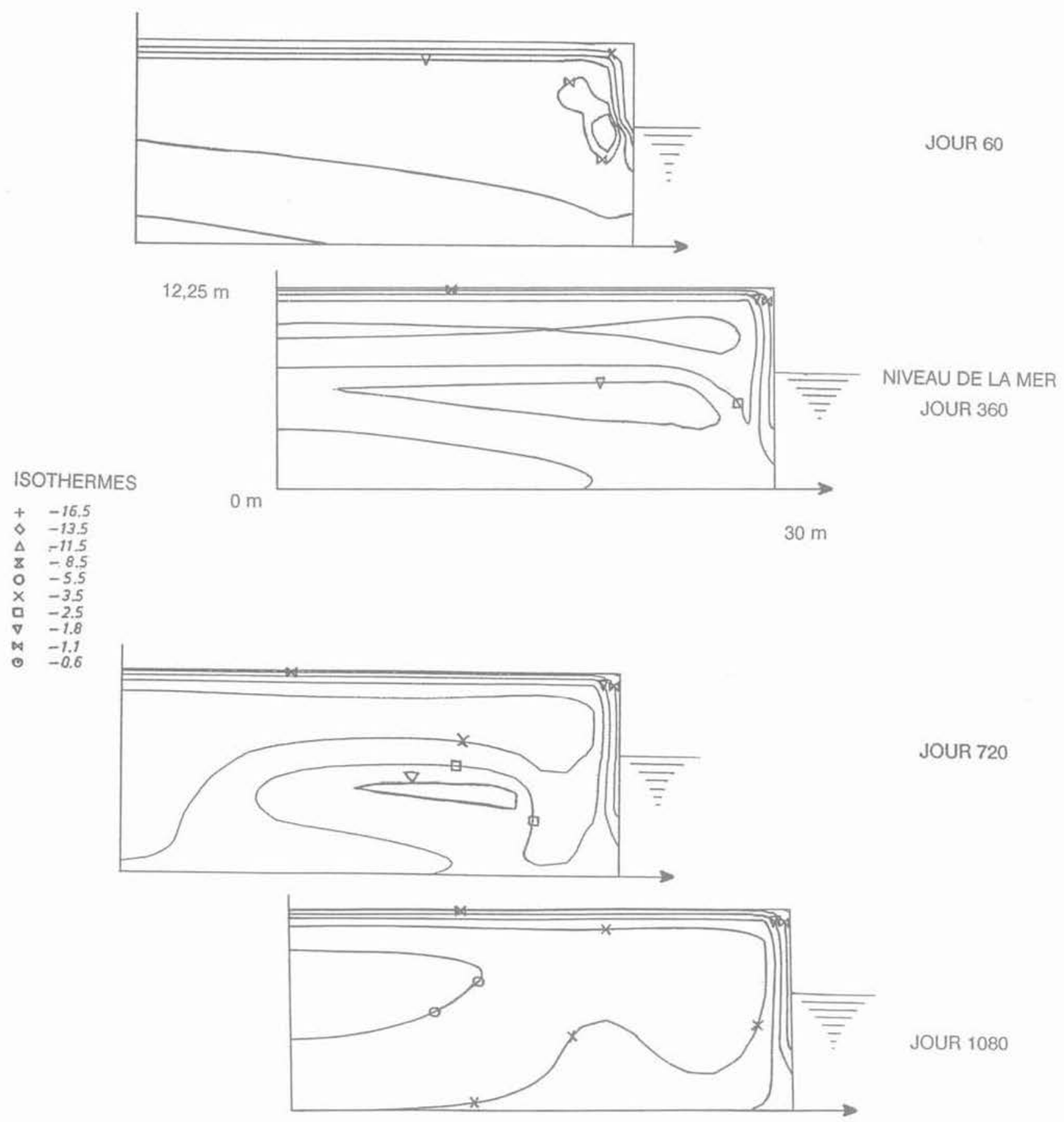

Fig. 4. - Isothermes pour la digue experimentale (LCPC).

Fig. 4. - Isotherms for the experimental breakwater (LCPC)

vection. La seconde précision qu'il faut apporter à ces calculs concerne la position du toit de la glace.

Par le calcul, on considère qu'il est situé au niveau de la mer alors que dans la réalité, il est situé à environ $2 \mathrm{~m}$ au-dessus de ce niveau. Ceci est probablement dû aux effets conjugués des marées, des vagues et à la dilatation lorsque l'eau se transforme en glace.

\section{Remblai Lion-Pollux}

Une série de calculs a été réalisée en considérant le substratum à $20 \mathrm{~m}$ sous le niveau de la mer. Nous avons modélisé la partie supérieure de louvrage en imposant la température de la mer à la côte - $7 \mathrm{~m}$ (les études sur le site ont montré qu'il n'y avait plus de glace à $3 \mathrm{~m}$ sous le niveau de la mer). Sous le niveau de la mer, il n'y a plus que des enrochements dont les caractéristiques sont celles de la couche centrale de la figure 3 .

Le calcul montre que l'épaisseur de la glace se stabilise après deux années. On peut donc penser que lorsque l'ouvrage sera terminé, une couche de glace d'environ $4 \mathrm{~m}$ d'épaisseur (deux sous le niveau 
moyen et deux au-dessus si on se refère à la digue expérimentale) aura pris place entre les blocs.

Sur la figure 5, on voit nettement l'influence de la mer. Au jour 720 , juste avant la débâcle estivale, à la vue de l'isotherme $-1,8^{\circ} \mathrm{C}$, on peut penser que la couche de glace s'épaissit vers le bas à proximité du talus. En effet, durant Ihiver, la banquise dont l'épaisseur va atteindre plus de $2 \mathrm{~m}$, impose des basses températures sur l'ouvrage (expérimentalement, on observe un gradient de température relativement constant entre la surface supérieure de la glace de mer à la température de l'air et la surface inférieure à la tempéraure de l'eau). En été, l'action réchauffante de la mer va diminuer cette épaisseur.
Signalons enfin que les mesures réalisées sur la digue expérimentale ont montré que les déplacements verticaux en surface étaient inférieurs à $2 \mathrm{~cm}$. L'ouvrage ne semble pas souffrir de la formation de cette glace qui paraît plutôt donner une cohésion au massif et le renforcer superficiellement.

\section{ACTION DE LA HOULE SUR L'OUVRAGE}

Dans cette région où la mer est gelée d'avril à décembre, la digue n'est soumise aux efforts de houle qu'en été. Si nous connaissons sa direction générale (Nord/Nord-Est), nous n'avons pas de mesures continues de sa hauteur et ne pouvons par conséquent

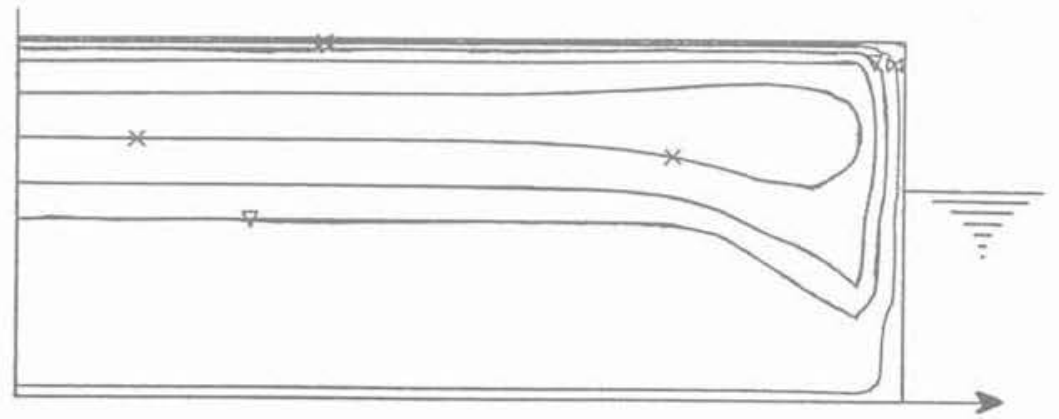

JOUR 720

ISOTHERMES

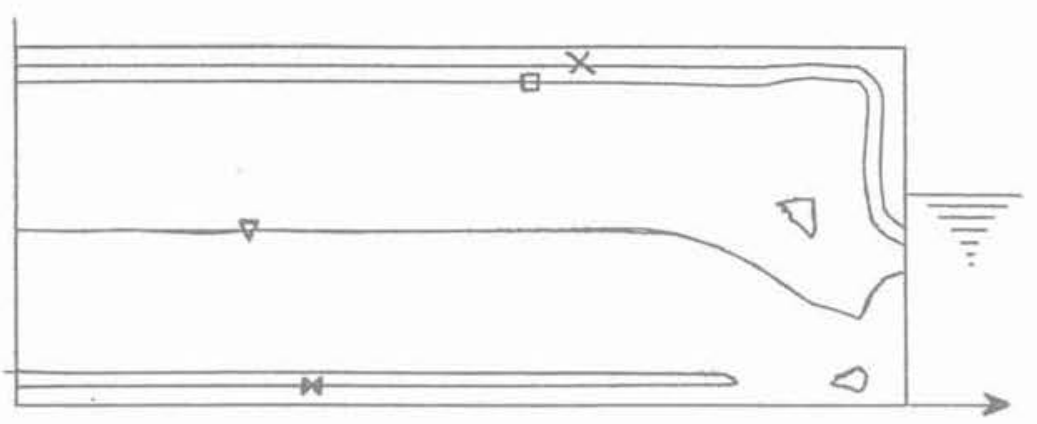

$+-16,5$

$\therefore \quad-13,5$

$\Delta \quad-11,5$

8 $-8,5$

o $-5,5$

$\times \quad-3,5$

ㅁ $-2,5$

$\nabla \quad-1,8$

a $-1,1$

JOUR 780

NIVEAU DE LA MER

JOUR 840

$12,25 \mathrm{~m}$

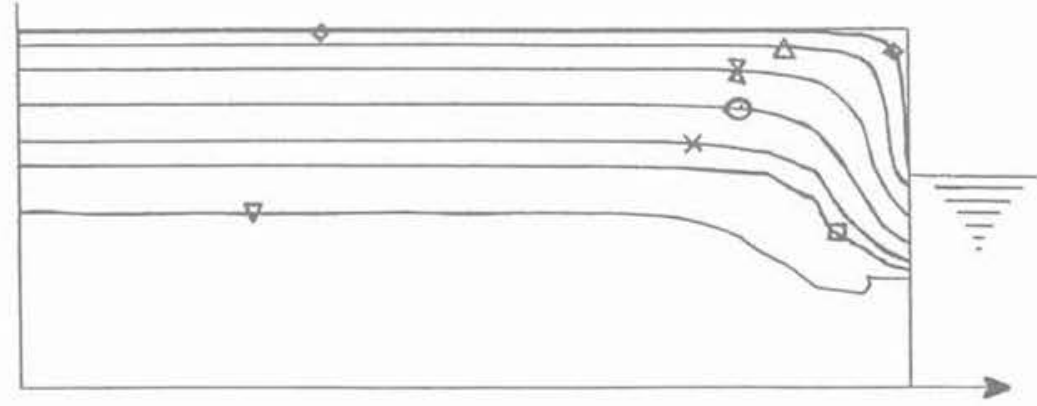

$0 \mathrm{~m}$

$30 \mathrm{~m}$

Fig. 5. - Action de la mer en été sur la digue Lion-Pollux (LCPC).

Fig. 5. - Action of seawater in summer on Lion-Pollux breakwater (LCPC). 
pas faire de traitement statistique permettant de calculer la houle de projet.

Des observations visuelles montrent que son amplitude n'excède pas $2 \mathrm{~m}$ et qu'elle ne se forme qu'en l'absence des vents dominants de Sud-Est. Les vagues sont une conséquence du vent sur la surface de la mer. Dans le cas des vents dominants qui viennent du continent, la distance sur laquelle elles peuvent se former, le Fetch, est de l'ordire de $1200 \mathrm{~m}$ (distance du continent à la digue). (Ceci constitue une valeur par excès car la bordure du continent est formée de falaises de glace de 25 ou $30 \mathrm{~m}$ de haut.

La formule de Sverdrup-Munk-Bretschneider permet de calculer la hauteur maximale significative $\mathrm{H}_{\mathrm{s}}$ :

$$
H_{s}=0,283 \frac{w^{2}}{g} \text { th }\left[0,0125\left(\frac{g^{F}}{w^{2}}\right)^{0,42}\right]
$$

où : $\mathrm{g}=9,81 \mathrm{~ms}^{-2}$

$w$ (vitesse du vent) $=83 \mathrm{~ms}^{-1}$ (vent maximal enregistré : $324 \mathrm{~km} / \mathrm{h}$ )

$$
\mathrm{F}(\text { Fetch })=800 \mathrm{~m} \mathrm{H} \quad \mathrm{H}_{\mathrm{s}}=2,6 \mathrm{~m} \text {. }
$$

La période correspondante, $\mathrm{T}_{\mathrm{s}}$ pour une houle de Stockes d'ordre 1, est de l'ordre de $3.3 \mathrm{~s}$. A ces valeurs, ont peut associer une hauteur de franchissement.

En utilisant les méthodes préconisées par le Shore Protection Manual [11], pour une pente de talus $\alpha=45^{\circ}$, on obtient :

$\mathrm{Ru}=0,6 \epsilon \mathrm{H}_{\mathrm{s}}$ avec $\epsilon=\tan (\alpha)\left[\frac{\mathrm{H}_{\mathrm{s}}}{1,56 \mathrm{~T}_{\mathrm{S}}^{2}}\right]^{-1 / 2}$

La hauteur de l'ouvrage a été fixée à $5 \mathrm{~m}$ auxquels il faudra ajouter les $50 \mathrm{~cm}$ de chaussée. La taille des blocs a été définie à partir de la formule de Hudson qui donne la masse minimale des enrochements de la carapace:

$$
w=\frac{\mathrm{H}_{\mathrm{s}}^{3} \rho}{\mathrm{Kd}(\delta-1)^{3} \cot (\alpha)}
$$

où $: \mathrm{Kd}=$ coefficient de stabilité

$\rho=$ masse volumique des blocs

$\delta$ (denstié des blocs) $=2,8$ et $\mathrm{Kd} \cong 4$ pour des enrochements anguleux.

La carapace est constituée de blocs de 2 à $3 \mathrm{t}$. Leur masse a été augmentée pour le musoir.

Le talus sud-ouest a la pente naturelle. Le talus nordest pouvant être soumis à des houles plus fortes que celles observées jusqu'à ce jour, une risberme de pente $3 / 1$ entre les côtes +2 et -2 devrait protéger la structure. Ces résultats sont à l'origine du dimensionnement de la digue expérimentale (ENGLER, LETAVERNIER, MEBAREK [3]).

\section{Gélifraction des blocs}

La stabilité de l'ouvrage peut être mise en danger si la masse des blocs n'est pas constante dans le temps. On sait que le froid peut avoir des effets indésirables sur certaines roches contenant de l'eau: écaillage pouvant aller jusqu'à l'éclatement.
Sur le site, les observations montrent que le gneiss ne semble pas produire de gélifracts mais ce n'est pas parce que le matériau en place n'est pas gélif que les blocs ne le sont pas. En effet, les massifs rocheux de l'archipel ne sont pas alimentés en eau alors que certains enrochements sont immergés. D'autre part, la porosité des blocs obtenus par minage pourrait être importante. Des essais ont donc été réalisés en laboratoire (LAUTRIDOU [8]).

Un simple examen de la porosité montre que les blocs sont a priori non gélifs : porosité $<0.5 \%$.

Pour confirmation, des échantillons saturés en eau douce et en eau salée ont été soumis à 330 cycles de gel et dégel $+15 /-25^{\circ} \mathrm{C}$. Aucune trace de gélifraction n'a été observée.

Pour s'assurer qu'une température très basse n'entraîne pas une augmentation de gélivité, des échantillons également saturés en eau douce et en eau de mer ont été soumis à 60 cycles de 2 jours $+15 /-50^{\circ} \mathrm{C}$ sans qu'aucune gélivité ne soit constatée. Le gneiss de Terre Adélie est particulièrement adapté à cet ouvrage.

\section{ACTION DE LA GLACE SUR L'OUVRAGE}

A partir du mois d'avril, la surface de la mer est généralement gelée et l'épaisseur de glace croit régulièrement (fig. 6). Si la glace qui se forme à l'intérieur de l'ouvrage participe à sa stabilité, il n'en est certainement pas de même pour la banquise qui exerce des efforts sur le talus.

\section{Efforts verticaux}

La glace accrochée à l'ouvrage peut se déplacer avec la marée et endommager le talus. On observe sur le terrain que, dès sa formation, elle suit le niveau de la mer. Une charnière se forme dès le début, désolidarise la banquise de la banquette (fig. 7) et de ce fait, les efforts verticaux sont limités.

\section{Efforts horizontaux}

Ils apparaissent essentiellement lorsque la glace vient se ruiner par compression. Les efforts sont limités par la résistance de la glace et sont donnés par la formule de KORZHAVIN [7] :

$$
\mathrm{F}=\mathrm{I} \cdot \mathrm{m} \cdot \mathrm{k} \cdot \sigma_{\mathrm{c}} \cdot \mathrm{D} \cdot \mathrm{t}
$$

où :

$F=$ force totale exercée sur la structure,

$\mathrm{I}$ = facteur d'indentation de P'ordre de 1 pour les structures larges,

$k=$ facteur de contact égal à 1 pour un contact parfait.

$\mathrm{m}=$ facteur de forme égal à 1 pour une structure cylindrique,

$\sigma_{\mathrm{c}}=$ résistance de la glace en compression simple,

$\mathrm{D}=$ largeur de la structure (on prendra $\mathrm{D}=100 \mathrm{~m}$, car les efforts sur les iles ne nous intéressent pas,

$\mathrm{t}=$ épaisseur de glace $(\mathrm{t}=1,5 \mathrm{~m})$.

Dans notre cas, $\frac{D}{t} \gg 1: m=1=1$. 
EPAISSEUR DE GLACE-DUMONT D'URVILLE

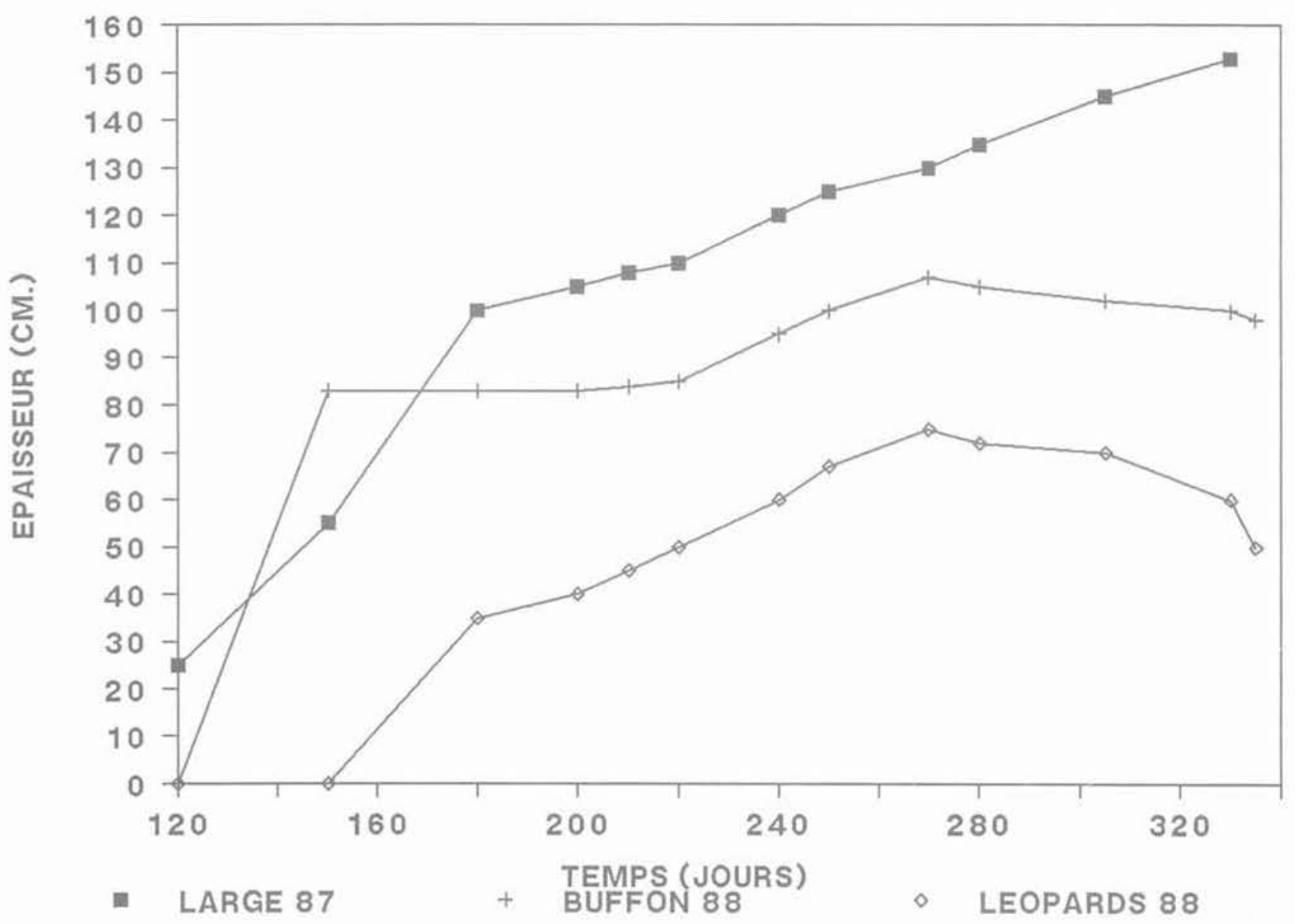

Fig. 6. - Epaisseur de la banquise en Terre Adélie (REGRETTIER, 1987; GUICHARD, 1988). Fig. 6. - Sea ice thickness in Terre Adelie IREGRETTIER, 1987; GUICHARD, 1988).

niveau moyen de la mer
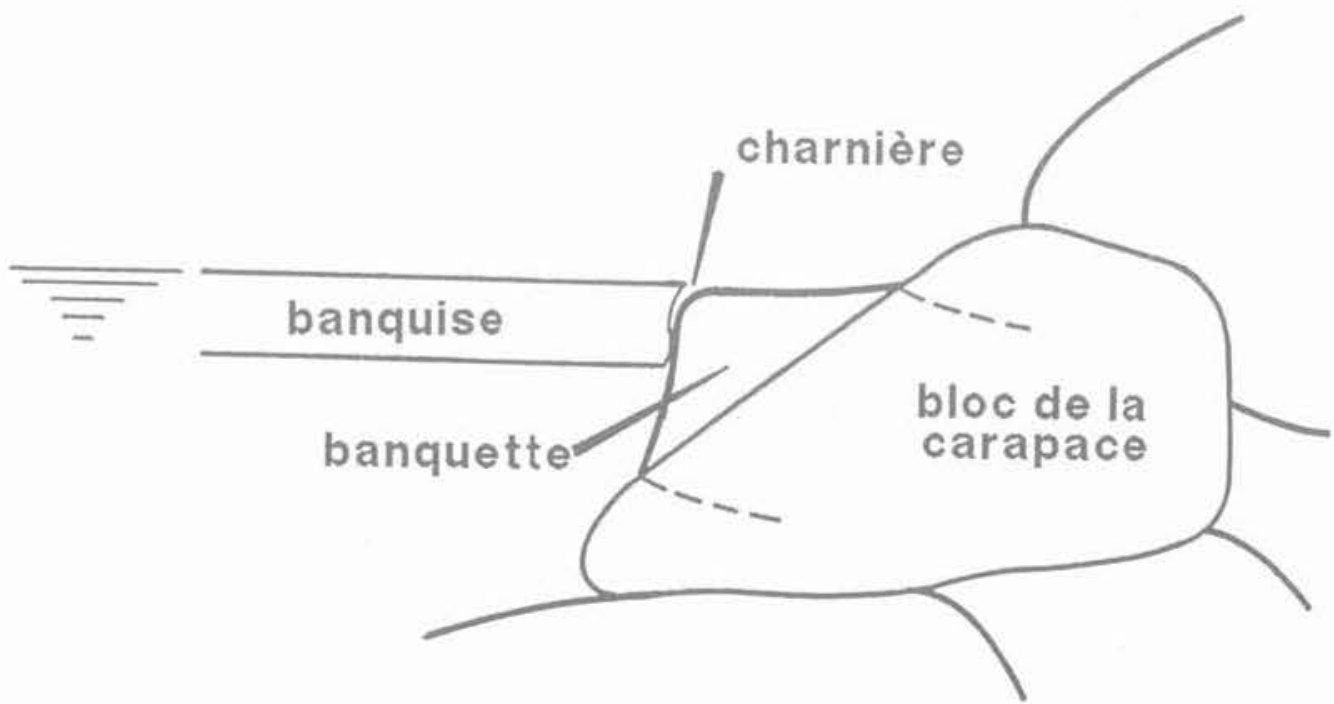

Fig. 7. - Mouvements verticaux engendrés par les variations du niveau de l'eau.

Fig. 7. - Uplifts of the ice sheet generated by rise in water level. 
Les ruptures non simultanées de la glace sont d'autant plus importantes que la surface de contact est grande. On peut raisonnablement prendre $\mathrm{k}=0.1$ (SANDERSON [10])

L'une des difficultés de cette méthode est de choisir $\sigma_{c}$ qui dépend de nombreux paramètres : température, salinité, âge, taille des grains, structure cristalline, taux de déformation. Une définition empirique du taux de déformation a été proposée par MiCHEL et TOUSSAINT [9] :

$$
\dot{\epsilon}=\frac{V}{4 D}
$$

où : $\mathrm{V}=$ vitesse de la glace .

En hiver, à Dumont d'Urville, la banquise est ancrée aux iles de l'archipel et sa vitesse est pratiquement nulle.

Soit $\mathrm{V}=1$ mètre $/$ mois :

$$
\dot{\epsilon} \cong 10^{-9} \mathrm{~s}^{-1}
$$

Dans ces conditions, $\sigma_{c} \cong 0,1 \mathrm{MPa}$ (fig. 8). La force résultante sur la structure est de l'ordre du megaNewton et la pression moyenne de la glace sur l'ouvrage est de lordre du kiloPascal.

En été, au moment de la débâcle, des morceaux de banquise peuvent venir heurter les talus sous l'effet du vent ou des courants.
Supposons une plaque de $1.5 \mathrm{~m}$ d'épaisseur, de largeur $50 \mathrm{~m}$ se déplaçant à $0,5 \mathrm{~ms}^{-1}$ :

$$
\dot{\epsilon} \cong 2,5 \cdot 10^{-3} \mathrm{~s}^{-1}
$$

La résistance en compression correspondante vaut $5,5 \mathrm{MPa} F \cong 40 \mathrm{MN}$ et la contrainte moyenne ou pression effective $\mathrm{P}=\frac{\mathrm{F}}{\mathrm{Dt}}$ est de l'ordre de $0.5 \mathrm{MPa}$.

Lorsque la glace se ruine en flexion sur un ouvrage incliné, une composante horizontale de l'effort agit sur la structure. Plusieurs modèles ont été proposés mais tous font intervenir un coefficient de frottement structure/glace que nous ne connaissons pas. De plus, ces efforts sont limités pour deux raisons:

- la résistance en flexion de la glace est généralement plus faible que sa résistance en compression : 0,1 à $0,8 \mathrm{MPa}$ (SANDERSON [10]) ;

- la flexion se produit principalement en début d'hiver, lorsque la glace de mer n'est pas trop épaisse.

La glace peut également se ruiner par flambement et dans ce cas encore, les efforts sont inférieurs à ceux associés à la ruine par compression.

\section{Chargements dynamiques}

En 1988, il a été montré par JEFFERIES et WRIGHT [6] que lorsque la glace vient se ruiner sur une structure, de fortes vibrations peuvent apparaître. Ces vibrations auraient pu être fatales au caisson « Molik-

\section{DUCTILE TRANSITION FRAGILE}

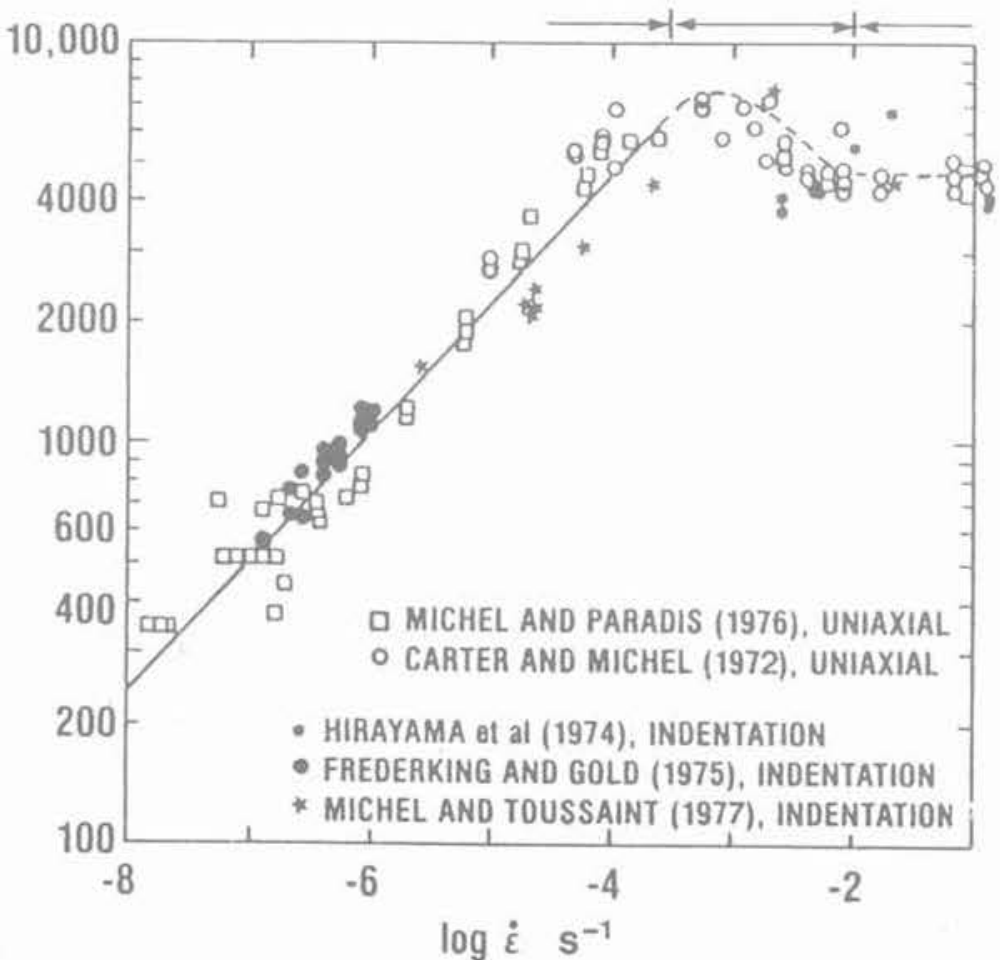

Fig. 8. - Résistance de la glace de mer en compression simple en fonction du taux de déformation $\dot{\varepsilon}$.

Fig. 8. - Unconfined, uniaxial crushing strength of sea ice as a function of strain rate $\epsilon$. 
paq " en mer de Beaufort : il s'agit d'un caisson d'acier de $100 \mathrm{~m}$ de diamètre, rempli de sable et posé sur une plate-forme sous marine également en sable. Sous l'effet des vibrations, la pression interstitielle a fortement augmenté, et la résistance au cisaillement fortement diminué alors que les pressions exercées par la glace sur le caisson étaient de $500 \mathrm{MN}$ ! Heureusement, le phénomène s'est arrêté rapidement en raison de la taille limitée de la plaque de glace.

La glace peut donc engendrer deux types d'action sur la digue : une pression sur une hauteur de $2 \mathrm{~m}$, sur une largeur donnée, et des vibrations.

Le risque essentiel lié aux vibrations est la liquéfaction du massif. Dans notre cas, on peut exclure ce risque pour deux raisons majeures:

- l'eau qui se trouve dans le massif est sous forme solide ;

- dans la frange non gelée à proximité des talus, la granulométrie est telle que ce phénomène semble peu probable (DAVIDOVICl [2]).

Pour la carapace, le volume des blocs est de l'ordre du $\mathrm{m}^{3}$ et pour le reste de l'ouvrage, la granulométrie est telle que $D_{50} \gg 1,5 \mathrm{~mm}$ et $\mathrm{Cu} \gg 15$, ce qui en fait un sol non liquéfiable.

Les efforts engendrés par la glace sur le talus pourraient entraîner une instabilité de l'ensemble (glissement de l'ouvrage sur le substratum), un endommagement local du talus, des cisaillements dans le remblai entre le niveau de la banquise et le substratum ou simplement un déplacement des blocs de la carapace.

Rien de cela n'a été observé sur la digue expérimentale depuis 1985. Les efforts exercés par la banquise en hiver ne sont pas suffisants pour provoquer lascension de morceaux de banquise sur les talus (ride-up), ou pour endommanger ces derniers. En ce qui concerne la stabilité de l'ensemble, elle est assurée d'une part par le frottement à la base sur le substratum, et d'autre part par les frottements latéraux des zones d'enrochements sur les îles.

\section{CONCLUSION}

La digue de Terre Adélie, malgré l'environnement polaire, bénéficie de conditions plutôt favorables. La transformation de l'eau en glace entre les blocs renforce l'ouvrage sans déformations dommageables et limite probablement les écoulements internes. Les enrochements produits sur le site ne sont pas gélifs.

Les vents dominants qui soufflent presque en permanence du continent ne disposent pas d'un fetch suffisant pour lever de fortes vagues, et de plus ils s'opposent à la houle qui vient du large. En hiver, les mouvements de banquise sont fortement réduits car la glace est ancrée aux différentes îles. Les efforts qu'elle exerce sur la structure sont faibles.

Depuis la construction de la digue expérimentale en 1985, aucun incident grave n'a été observé. Le climat sévère de l'Antarctique ne paraît pas nuire à la tenue de l'ouvrage et il semble aujourd'hui que le risque le plus important soit lié à une houle du nord exceptionnelle.

\section{REMERCIEMENTS}

L'auteur remercie particulièrement le territoire de Terres Australes et Antarctiques Françaises sans lequel ces études n'auraient pu être réalisées ainsi que $\mathrm{M}$. A. DUPAS, du Laboratoire Central des Ponts et Chaussées.

\section{BIBLIOGRAPHIE}

[1] ANDERSLAND O.B., ANDERSON D.M. (1978), Geotechnical Engineering for cold regions. Mac Graw Hill.

[2] DAVIDOVICI V. (1985), Génie parasismique. Presses de l'ENPC

[3] ENGLER M., LETAVERNIER G., MEBAREK A. (1987), Caractérisation des températures dans les structures continues et discontinues en milieu polaire. Rapport interne, Territoire des Terres Australes et Antarctiques Françaises.

[4] ENGLER M., REGRETTIER J.F. (1989), Adelie land airport, 8th international conference on Offshore Mechanics and Arctic Engineering - La Haye.

[5] ETTEMA R., KENNEDY J.F. (1982), Ice study for the port of Nome, Alaska-lowa Institute of Hydraulic Research, Technical Report \# 260.

[6] JEFFERIES M.G., WRIGHT W.H. (1988), Dynamic response of "Molikpag" to ice. structure interaction, 7th international conference on Offshore Mechanics and Arctic Engineering - Houston.

[7] KORZHAVIN K.N. (1982), Action of ice on engineering structure. Traduit du russe par le Cold Regions Research Engineering Laboratory - Hanover, New Hampshire.

[8] LAUTRIDOU J.P. (1989), Gélivité des blocs du remblai de Terre Adélie sous l'influence des changements de température et en présence de sels marins. Centre de Géomorphologie du CNRS, Caen.

[9] MICHEL B., TOUSSAINT N. (1977), Mechanisms and Theory of indentation of ice plates. Journal of Glaciology (9).

[10] SANDERSON T.J.O, (1988), Ice mechanics, Risk to offshore structures. Graham \& Trotman.

[11] Shore Protection manual (1984), Coastal Engineering Research Center US Army corps of engineers, Washington D.C. 